RESEARCH HIGHLIGHTS

\title{
On the verge of collapse
}

Several microtubule-disrupting agents that are used in cancer therapy, such as colchicine and combretastatin A4 phosphate (CA4P), also have interesting additional antitumour effects, such as causing rapid and catastrophic shutdown of the tumour vasculature. The mechanism of these effects has been unclear, but Shahin Rafii and colleagues report that $\mathrm{CA} 4 \mathrm{P}$ induces the regression of unstable tumour neovessels by disrupting the endothelial cell junction molecule vascular endothelial cadherin (VE-cadherin).

CA4P is a stilbene compound that was isolated from a South African bush willow, Combretum caffrum. CA4P binds tubulin with a higher efficacy than colchicine, and was therefore initially investigated as an anti-mitotic agent. However, it was later observed to also induce vascular shutdown and necrosis in tumours. Clinical trials have revealed its positive effects, either as a single agent or in combination with chemotherapy, in patients with ovarian, lung or anaplastic thyroid cancer.

Rafii's group evaluated the effects of this compound on the neoangiogenic endothelial cells that form blood vessels during tumour angiogenesis. They found that at low, non-toxic doses, CA4P not only inhibited the proliferation of endothelial cells, but also disrupted cell-cell junctions, cell migration and anchorage, resulting in cell death.

VE-cadherin is an important mediator of cell-cell contacts and cytoskeletal organization, so the authors investigated its role in the endothelial cell's response to this drug. After only 3-6 hours of exposure to CA4P, VE-cadherin and its signalling partner $\beta$-catenin, which normally localize to cell-cell contacts, were redistributed, and by 18 hours, cell contacts were lost. Stabilization of the VE-cadherin complex through expression of the adenovirus $E 4$ gene protected cells against the effects of CA4P, indicating that disruption of this complex is an important mechanism of this drug. Biochemical analyses revealed that CA4P rapidly diminished the tyrosine phosphorylation of VE-cadherin and $\beta$-catenin, thereby blocking the endothelial signalling pathway that is necessary for maintaining a functional endothelial cell structure and survival.

So, what effects does this drug have on established vessels? Threedimensional tube formation assays revealed that CA4P blocks not only the formation of a capillary tube network, but could destabilize a preestablished endothelial network. This only occurred, however, in the absence of the smooth muscle cells that normally stabilize vessels. Furthermore, in vivo studies showed that CA4P caused tumour necrosis and the loss of the tumour vasculature in mouse models.

Rafii's group proposes a model in which normal blood vessels, which are lined with smooth muscle cells, are stable and protected against CA4P. Because nascent unstable tumour neovessels are not ensheathed by the smooth muscle cells, they are selectively destabilized CA4P through its disruption of VE-cadherin-mediated cell-cell contacts. This drug could therefore be used as a tumourspecific agent with low levels of toxicity to the normal vasculature - clinical trials that are underway have shown this to be the case.

Further studies are required to determine whether CA4P functions by directly disrupting the VE-cadherin- $\beta$-catenin complex, or how it might indirectly alter the phosphorylation and signalling of this complex, perhaps by interfering with cytoskeletal reorganization in endothelial cells.

Kristine Novak (1) References and links ORIGINAL RESEARCH PAPER Vincent, L. et al. Combretastain A4 phosphate induces rapid regression of tumor neovessels and growth through interference with vascular endothelialcadherin signaling. J. Clin. Invest. 6 October 2005 (doi: $10.1172 / \mathrm{JCl} 24586)$

FURTHER READING

Rafii, S. et al. Vascular and haematopoietic stem cells: novel targets for anti-angiogenesis therapy? Nature Rev. Cancer 2, 826-835 (2002)

\section{IN BRIEF}

\author{
METHYLATION
}

\section{MGMT promoter methylation and field defect in sporadic colorectal cancer.}

Shen, L. et al. J. Natl Cancer Inst. 97, 1330-1330 (2005)

Multiple independent tumours can arise in a particular tissue. In inherited or carcinogen-induced tumours this can be explained by an underlying shared defect in the cells (a field defect), but it is unclear how it occurs in sporadic tumours. In half of the patients with sporadic colorectal cancer in this study, there was inactivation in tumours of the $\mathrm{O}^{6}$-methylguanine-DNA methyltransferase (MGMT) gene by methylation and, in most cases, in the adjacent normal-looking tissue as well. This implicates MGMT methylation as the field defect.

\section{CANCER GENETICS}

Amplification of a chromatin remodeling gene, Rsf-1/HBXAP, in ovarian carcinoma.

Shih, I. M. et al. Proc. Natl Acad. Sci. USA 102, 14004-14009 (2005)

Through digital karyotyping and fluorescence in situ hybridization analysis, Shih et al. found that chromosome 11q13.5 was amplified in high-grade ovarian carcinoma samples. They also identified a potential oncogene at this locus, RSF1, which was overexpressed in ovarian tumours with this amplification. Patients with tumours that overexpressed this gene had shorter survival times than those without the amplification, and overexpression of RSF1 was sufficient to transform non-neoplastic cells.

\section{IMMUNOTHERAPY}

Tumor-targeted, systemic delivery of therapeutic viral vectors using hitchhiking on antigen-specific T cells.

Cole, C. et al. Nature Med. 11, 1073-1081 (2005)

Antigen-specific $\mathrm{T}$ cells circulate freely within the body and accumulate specifically at sites of antigen expression, such as tumours. Using this knowledge, combined with the fact that retroviral particles adhere non-specifically to T cells, the authors successfully delivered therapeutic viruses to tumour cell in a mouse model. Retroviral vectors expressing interleukin- 12 or Herpes Simplex virus thymidine kinase caused the regression of metastatic masses in vivo. This approach might prove useful in fully immunocompetent patients.

\section{CANCER GENETICS}

\section{FASL-844C polymorphism is associated with} increased activation-induced T cell death and risk of cervical cancer.

Sun, T. et al. J. Exp. Med. 3 October 2005 (doi: 10.1084/jem.20050707)

Functional polymorphisms in FAS and FAS ligand (FASL) are associated with susceptibility to certain cancers. Sun et al. found that expression of the gene variant FASL- $844 \mathrm{C}$ by T cells increases their levels of activation-induced cell death. The authors observed a threefold increased risk of cervical cancer among subjects with the FASL-844C genotype. They propose that this increased risk is due to activation-induced cell death of tumour-specific T cells, resulting in immune-response evasion by the tumour. 